\title{
24-hour nursed care for people with severe and enduring mental illness
}

\author{
Frank Holloway
}

This report (NHSE, 1996a), produced by a consultancy firm, was published in February 1996 as part of a package of measures to improve mental health services, promoted by the Secretary of State for Health, which included a promised $\$ 95$ million additional funding for 1996/7 (Court, 1996). It should be read in conjunction with a short but important publication from the Department of Health (DoH) on "the spectrum of care - a summary of comprehensive local services for people with mental health problems" (DoH, 1996) and a "checklist' for purchasers on 24-hour nursed care developed by the consultancy with the help of a $\mathrm{DoH}$ steering group including four psychiatrists (Whittaker \& Welch, 1996). Together these documents represent a very welcome attempt at encouraging informed purchasing of mental illness services while simultaneously ensuring that both purchasers and potential providers have a clear understanding of the priorities of the centre (NHSE, 1996b).

The term '24-hour nursed care' will be unfamiliar to clinicians. Analysis of the relevant texts suggests that it is synonymous with the 'hospital hostel' or 'hostel ward' (DoH, 1996. 3.18; Reid \& Garety, 1996), which the DoH has long advocated (DoH, 1991), with the significant proviso that any new development will be commissioned within the mixed economy of care (NHSE, 1996a, 3.3). Providers might therefore include the private and voluntary sector as well as the local NHS Trust(s). The policy context is complex but includes continuing Governmental commitment to the mental hospital closure programme and community care; "moral panic" over the alleged consequences of community care (Holloway, 1996); political and legal difficulties over the role of the NHS in the provision of continuing care (NHSE, 1995), which reflect problems that we as a society face in the funding of long-term care of elderly people; a slow collapse in the capacity of Soclal Service Departments to fund expensive residential and nursing home placements for the severely mentally ill; and the implications of a clearly signalled intention to reduce the use of maximum secure provision (the Special Hospitals) despite the current failure to develop long-stay medium security within the NHS.

Costings for 24 hour nursed care facilities are provided: at between $\$ 25$ and $\$ 50000$ per place these appear reasonable, with some generosity about the need for support staff if residents are truly to participate in their care. Economies of scale are realistically identified (20 residents can be cared for at much the same cost as 8). A service model is implied in the quality standards appended to the original paper (NHSE, 1996a) and clarified in the checklist for purchasers (Whittaker \& Welch, 1996). The issue of meaningful occupation for residents, always a problem for hospital and residential care, is largely ignored.

The paper attempts to identify the potential client group for 24-hour nursed care, which is not clearly defined but includes as a matter of urgency the "new long stay" (NHSE, 1996a, 1.6). Unusually, for DoH publications, outline minimum figures of need are offered (10 beds per 100000 total population) with the accurate proviso that the prevalence of psychosis may be very much higher in certain inner urban areas. Significantly there is great confusion about the relationship between any unit and the mainstream mental health services. Locally we have got over this by offering dedicated consultant, psychologist and occupational therapist time from our Trust to a 24 hour nursed care unit run by a voluntary sector provider, with the agreement that any readmission (an uncommon phenomenon) is to the relevant sector service. This facility was set up long before the clumsy neologism of 24-hour nursed care was dertved and appears to function well, albeit requiring continual monitoring from Trust staff.

There is an undoubted lack of 24-hour staffed residential care targeted at the most severely mentally ill. However, there are difficulties with the 24-hour nursed care proposals. Flrstly, the levels of local need for such provision are unknown. The most recent national prevalence 


\section{EDITORIALS}

figures for the new long stay (6.1 per 100000 population (Lelliott \& Wing, 1994) are significantly lower than the proposed levels of 24-hour nursed care beds, which are supposed to address the new long stay problem. It is clear that these patients are a very heterogeneous group with a wide variety of long-term residential care needs, of which the commonest will be staffed residential rather than nursed care and the second commonest long-term medium secure care (Holloway \& Wykes, 1996). Thus any service developments would have to be based on a very thorough local assessment of need. Secondly, to avoid becoming a very expensive dumping ground within the local service system, any 24-hour nursed care unit would have to be integrated into the mainstream psychiatric services, with dedicated sessions from a full multidisciplinary team. It would also have to be subject to pressures to ensure that, as appropriate, residents could move on to less supported and more normalising care environments. Thirdly, there is the crude issue of money. Each 24-hour nursed place will be equivalent to the cost of one or two experienced community nurses, occupational therapists or psychologists or a yearly supply of a novel antipsychotic for 10 or 20 patients. If the purchasers identify 24 hour nursed care as a priority, they will have to ensure that its development is not at the expense of local acute and community mental health services (what provider would cope with 50 less CPNs?). There is no current evidence in favour of the optimistic, indeed implausible, Audit Commission view that costs can be offset against reductions in acute hospital services (NHSE, $1996 a, 3.1)$, although 24-hour nursed care beds might very appropriately be developed in the context of a hospital closure programme or if real money became available for mental health service developments.

\section{References}

Courr, C. (1996) Government admits mental health service problems. British Medical Journal, 312, 531-532.

DEPARTMENT OF HEALTH (1991) Residential Needs for Severely Disabled Psychiatric Patlents: The Case for Hospital Hostels. London: DoH.

- (1996) The Spectrum of Health Local Services for People with Mental Health Problems. London: DoH.

HowOWAY, F. (1996) Community psychiatric care: from libertarianism to coercion. Moral panic and mental health policy in Britain. Health Care Analysis, 4. 235243.

- \& WYkes, T. (1996) New Long Stay Patients: Who are They and what are Their Needs? Paper presented at the 11 th TAPS Conference, London.

LELuOT, P. \& WnG, J. (1994) A national audit of new longstay psychiatric patients. II: Impact on services. British Joumal of Psychiatry, 166, 170-178.

NATIONAL HEALTH SERVICE EXECUTIVE (1995) NHS Responsibilities for Meeting Continuing Health Care Needs. HSG (95)8. Leeds: NHSE.

- (1996a) 24 Hour Nursed Care for People with Severe and Enduring Mental Illness. Leeds: NHSE.

- (1996b) Prlorities and Planning Guidance for the NHS: 1997/8. Leeds: NHSE.

REID, Y. \& GARETY, P. (1996) A hostel-ward for new long stay patients: Sixteen years' progress. Journal of Mental Health, 6, 77-89.

WHITTAKER, N. \& WELCH, P. (1996) Commissioning 24 Hour Nursed Care for People with Severe and Enduring Mental Illness. A Practical Checklist for Purchasers. Leeds: NHSE.

Frank Holloway, Consultant Psychiatrist, Maudsley Hospital, Denmark Hill, London SE5 8AZ 